\title{
Recent advances in applications of multimodal ultrasound-guided photoacoustic imaging technology
}

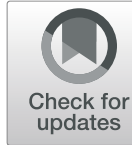

\author{
Shanshan Wang ${ }^{1 *}$, Yunfeng Zhao ${ }^{2}$ and Ye Xu
}

\begin{abstract}
Photoacoustic imaging (PAI) is often performed simultaneously with ultrasound imaging and can provide functional and cellular information regarding the tissues in the anatomical markers of the imaging. This paper describes in detail the basic principles of photoacoustic/ultrasound (PA/US) imaging and its application in recent years. It includes near-infrared-region PA, photothermal, photodynamic, and multimode imaging techniques. Particular attention is given to the relationship between PAI and ultrasonic imaging; the latest high-frequency PA/US imaging of small animals, which involves not only B-mode, but also color Doppler mode, power Doppler mode, and nonlinear imaging mode; the ultrasonic model combined with PAl, including the formation of multimodal imaging; the preclinical imaging methods; and the most effective detection methods for clinical research for the future.
\end{abstract}

Keywords: Photoacoustic/ultrasound imaging, The second near-infrared photoacoustic, Photothermal therapy, Photodynamic therapy, Multi-mode imaging

\section{Photoacoustic/ultrasound imaging}

Photoacoustic imaging (PAI) is a new imaging method that combines the high contrast of optical imaging with the spatial resolution and penetration depth of ultrasound (US). The basic principle of this imaging can be simply summarized as imaging by detecting broadband ultrasonic waves excited by pulsed light. A pulsed laser (usually a nanosecond pulse) irradiates a sample, which absorbs the laser energy that is further translated into heat energy, leading to transient thermal expansion of the sample and a high-frequency mechanical pressure wave (i.e., ultrasonic). The US wave travels through the medium of transmission to the surface, such as in an US transducer, which is processed by a computer, and a photoacoustic (PA) image is obtained [1]. In addition, the similarity between PA and ultrasonic imaging methods enables the integration of the two modes, and

\footnotetext{
* Correspondence: shanshan.a.wang@fujifilm.com

'VisualSonics Business Department, FUJIFILM (China) Investment Co. Ltd., Beijing 100026, China

Full list of author information is available at the end of the article
}

there is a good synergy that confers the ability to visualize morphology, function, and molecular characteristics. Based on recent developments, an updated PA/US imaging system has integrated all US modes (B-mode, Doppler mode, contrast mode, etc.) into PAI [2]. Usually, PAI alone cannot provide appropriate anatomical information for the effective localization of PAI signals in animals. Although the method of white light superposition is convenient, the repeated imaging of the same animal at different time points often leads to misinterpretation of the signal positioning because of the difficulty of repositioning the animal [3]. Moreover, PA/US can account for the limitations and deficiency of the single PA model to improve the accuracy of early detection of diseases [4]. Vevo LAZR-X (Fujifilm Visualsonics, Fig. 1), a commercial US and PA multi-mode imaging system, interprets PA signals regarding anatomical structure information [5]. The Vevo LAZR-X uses ultra-highfrequency electronic linear probes, with a maximum characteristic high-frequency US detector (> $20 \mathrm{MHz}$ ), where high-frequency technology is not only applied in 


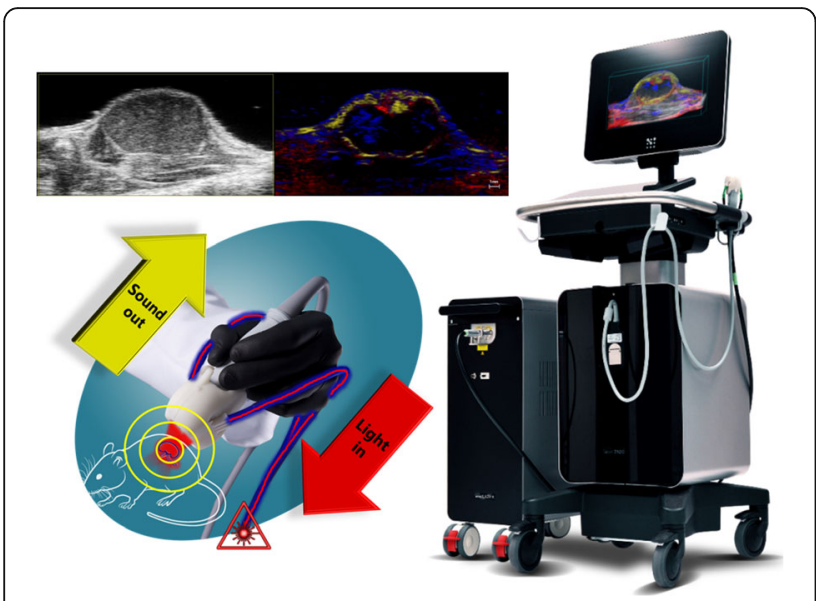

Fig. 1 Schematic of PA/US multimode imaging system

the ultrasonic system, but also in the PA system [6]. High frequency leads to ultra-high anatomical resolution, and the high acquisition frequency of the system can complete instantaneous imaging to make real-time dynamic images smooth and clear, to obtain anatomical images, hemodynamic details, and accurate positioning [7-9]. PA/US multimodal imaging can be used to obtain anatomical and physiological information. This system has been used in various preclinical studies, such as tumor detection and staging, neuroscience research, reproductive development research, in vivo stem cell tracing, lymph nodes, and thrombosis [10-12]. In clinical application, PA/US has been used to acquire images of a vasculature mimicking phantom, a contrast-enhanced rat GI tract in vivo, and a human forearm in vivo. This developed system is the first PA/US system based on a conventional clinical US machine and provides conveniences such as handheld operation, an intuitive user interface, complete portability, and real-time imaging [13]. In 2015, Garcia-Uribe et al. [14] developed a dualmodality PA and US imaging system to noninvasively detect sentinel lymph nodes based on the accumulation of methylene blue dye. Ultimately, they aimed to guide percutaneous needle biopsies and provide a minimally invasive method for axillary staging of breast cancer. In 2014, Daoudi et al. [15] reported a system that is a first step toward an affordable portable combined US-PA imaging system. The system performance was tested in vitro and in vivo by imaging a human finger joint. In 2015, Sun et al. [16] reported that a Vevo LAZR Fujifilm Visualsonics (high-frequency small animal PA/US imaging system) was used to investigate whether prophylactic edaradone (a free radical scavenger used to treat ischemic stroke in Japan) could prevent infarction and cognitive impairment in transient cerebral hypoxicischemic models in mice. Blood oxygen saturation $\left(\mathrm{StO}_{2}\right)$ and blood flow were detected in the cerebral cortex. A schematic diagram of the transient hypoxiaischemia (tHI) model. MCA/ICA/ECA, middle, internal, and external carotid artery. RCCA, the right common carotid artery (Fig. 2a). Doppler flow imaging and 3D reconstruction showed reduced perfusion in the ipsilateral hemisphere and the reversal of blood flow in the right internal carotid artery (RICA) in the Circle of Willis after right carotid artery occlusion (RCCAO) (Fig. 2b). PAI showed that $\mathrm{StO}_{2}$ in the ipsilateral cortex was reduced from $\sim 70 \%$ to $\sim 61 \%$ upon $\mathrm{RCCAO}$ and plummeted to $\sim 20 \%$ upon exposure to hypoxic air (Fig. 2c). After $30 \mathrm{~min}$ tHI, the ipsilateral hemisphere showed poorer recovery of $\mathrm{StO}_{2}$ than the contralateral cortex ( $28 \%$ compared with $\sim 39 \%$ in normoxia and $~$ $53 \%$ vs $\sim 70 \%$ under $100 \%$ oxygen) (Fig. $2 \mathrm{~d}) \quad(n>3$ times). This pattern suggests that a transient episode of HI may impair subsequent cortical oxygenation. In 2018, Qin et al. [17] demonstrated that a semiconductor polymer (SP) combined with the PA properties of nanoparticles can trace these cells in mice. This is achieved by virtue of two benefits of the photoacoustic properties of nanoparticles (PANPs). First, strong PA signals and specific spectral features of SPs allow PAI to sensitively detect and distinguish a small number of PANP-labeled cells (2000) from background tissues in vivo. Second, the PANPs show a high efficiency for human embryonic stem cell-cardio myocytes (hESC-CMs) labeling without adverse effects on cell structure, function, and gene expression. US imaging can assist in the transplantation of these cells and can be used to evaluate cardiac repair therapy with high-resolution photosonography. PAI and US imaging were also performed to visualize the engraftment of transplanted cells in vivo. Figure 3a shows an ultrasound image of cardiac structures in a short-axis view, in which the injecting needle is difficult to be differentiated from the ultrasound image due to its duplicated artifacts and similar intensity to the tissues. An electrocardiogram and respiratory coupling were used to acquire B-mode US images and multi-spectral PA images from short-axis (Fig. 3c) and long-axis views (Fig. 3e). Their merged images in Fig. 3d and $f$ showed the 3D spatial relationship between the transplanted cells and the host myocardium at a high resolution $(\sim 100 \mu \mathrm{m})$. In addition, the PANP-labeled cells also emitted a nearinfrared fluorescence upon laser excitation (peak at 820 $\mathrm{nm}$ ), which facilitated FI to further confirm the cell transplantation in vivo (Fig. 3b).

\section{PAI in the second near-infrared window (the second near-infrared $P A$ )}

The advantages of the second near-infrared (NIR-II) PAI are as follows: large penetration depth, low background noise, high maximum allowable irradiation energy and less absorption and scattering by skin tissues, which is 


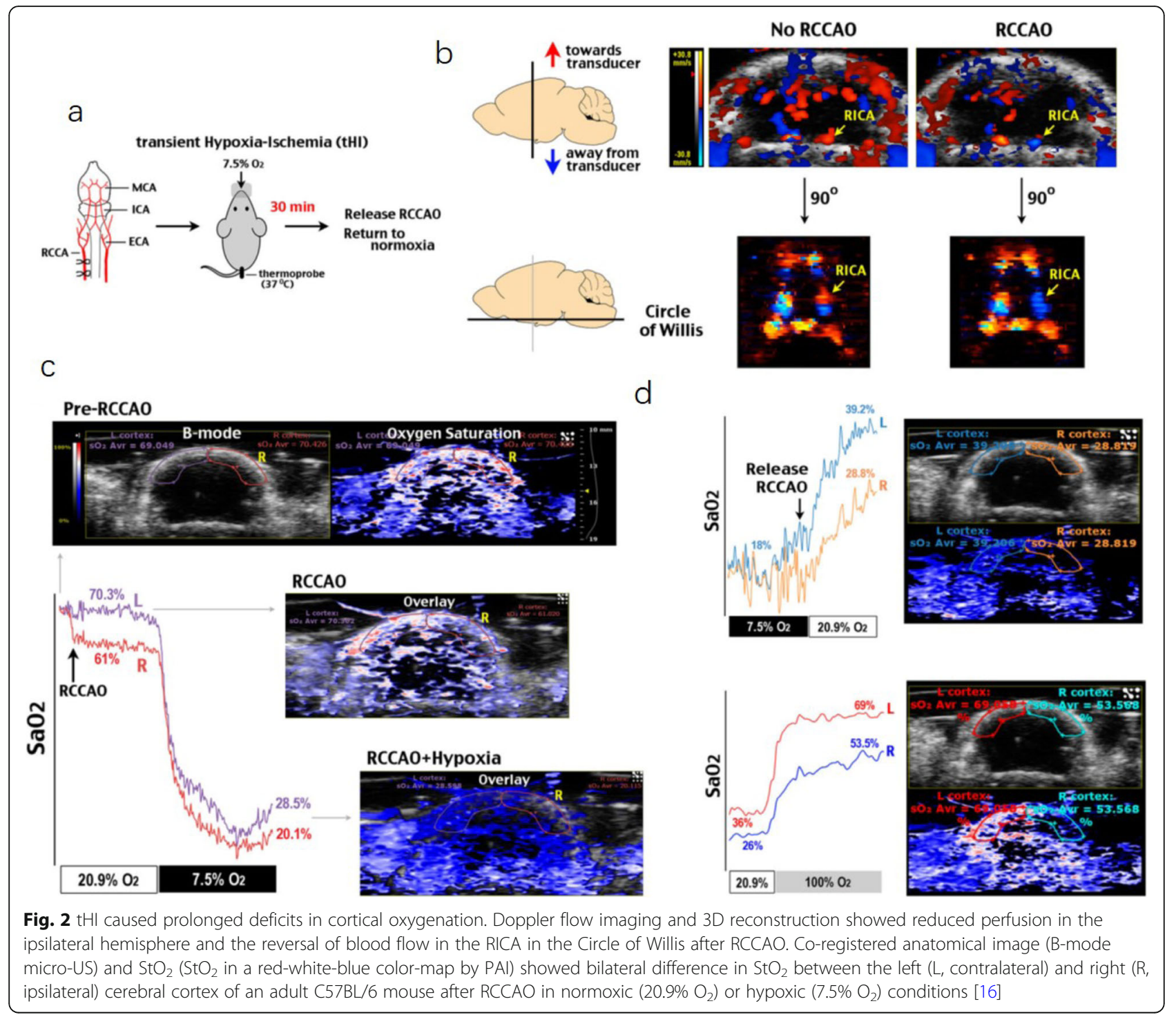

conducive to achieving high-resolution imaging of deep tissues [18]. However, melanin, hemoglobin, and other biological components absorb and scatter light in the first near-infrared $(650-970 \mathrm{~nm})$ window, resulting in background interference and spontaneous fluorescence, which can reduce the sensitivity, spatial resolution, and contrast of PAI. In contrast, PAI has less spontaneous fluorescence interference in the display of NIR-II (1200$2000 \mathrm{~nm}$ ) window and no background signal. Because of the lower light absorption and scattering of endogenous biomolecules in this area, it has higher sensitivity and spatial resolution and deeper tissue penetration depth. Endogenous PAI contrast agents in NIR-II include fat, collagen, and other components [19]. An external PAI contrast agent in NIR-II can locally enhance tissue absorption performance, enhance the PA signal, and improve image contrast. Therefore, the use of an external contrast agent in NIR-II is an important condition for deep tissue PAI [20].

However, because of a lack of NIR-II absorbing contrast agents, PAI is most often carried out in the NIR-I window [21]. In 2019, Zhang et al. [22] reported semiconducting polymer nanoparticles (SPNs) composed of DPP-based two-acceptor semiconducting polymers with strong electron-deficient acceptors (Benzobisthiadiazole) to develop an efficient NIR-II PAI/photothermal therapy (PTT) agent. With high photothermal conversion efficiency (60\%), SPNs3 exhibited strong PA signals at 1280 $\mathrm{nm}$ that were detected by commercial NIR-II PAI systems (Vevo LAZR-X), and PAI-imaging guided photothermal tumor therapy was realized in live animals under NIR-II light excitation. The PA intensity generated by SPNs3 at $1280 \mathrm{~nm}$ was 2.2-fold stronger than that of SPNs2 at $1200 \mathrm{~nm}$. The PA signal of SPNs3 was 
a

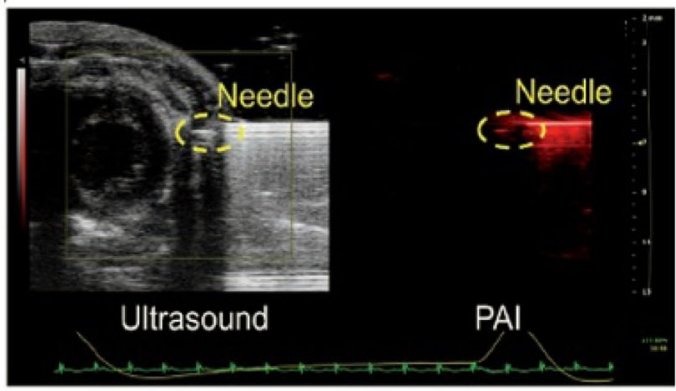

C

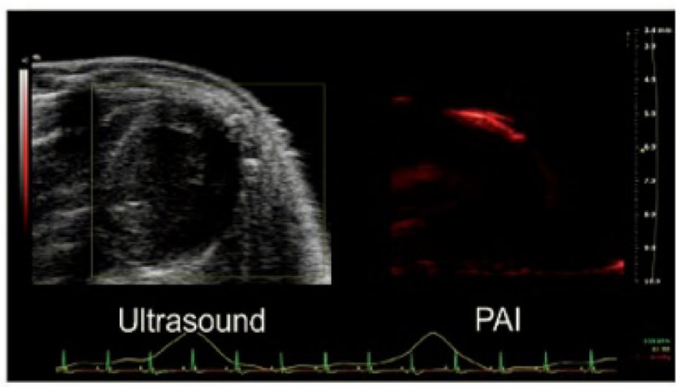

d

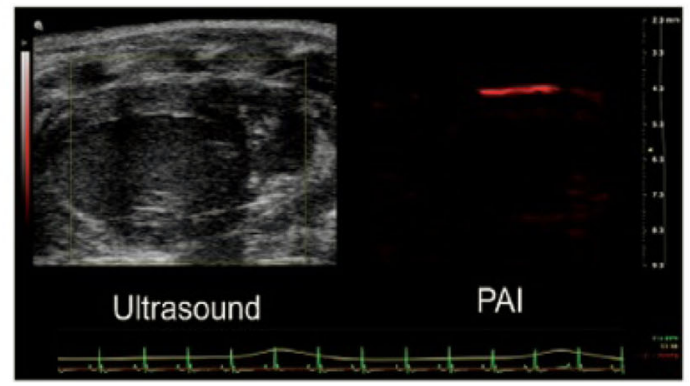

b

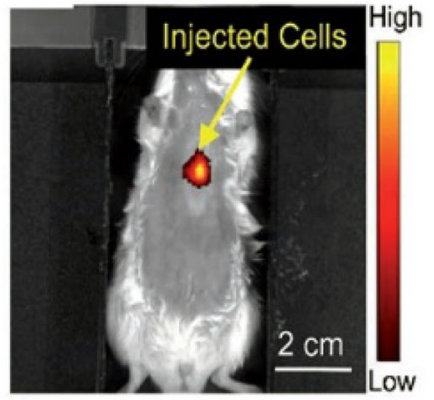

e

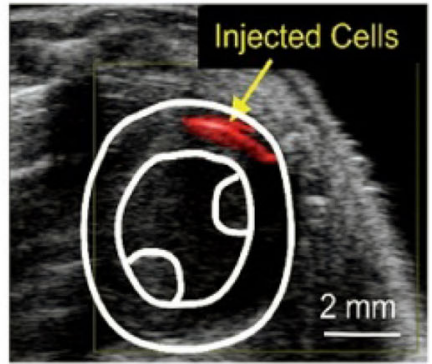

f

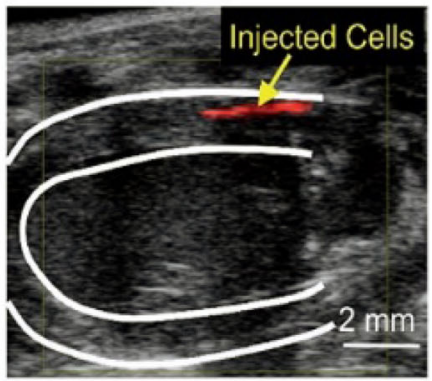

Fig. 3 PAl of the injection and engraftment of PANP-labeled hESC-CMs in mouse hearts in vivo [17]

$23 \sim 27$-fold higher than that of FBS and PBS at 1280 $\mathrm{nm}$, which could indicate an excellent PA signal-tonoise ratio in vivo. As depicted in Fig. 4a, the maximum PA signals of SPNs2-3 were observed at approximately 1200 and $1280 \mathrm{~nm}$ in the NIR-II PA spectra. In Fig. 4b, SPNs2-3 exhibit a good linear relationship between the PA signal intensity and concentration. After intratumoral injection of SPNs2-3 (Fig. 4c), from Fig. 4d, we found that the NIR-II PA signals of the SPNs3 solution were much higher than those of SPNs2 under chicken tissue of different thickness, exhibiting the good deeptissue PA imaging capabilities of SPNs3. relatively stronger PA signals for SPNs3 were detected in the tumor region compared with those in the untreated group and the SPNs2-treated group, implying that SPNs3 is an available PA agent in the NIR-II window. In 2020, Zhu et al. [23] developed dual biologically responsive nanogapped gold nanoparticle (Au NPs) vesicles loaded with immune inhibitors and carried anticancer polymeric prodrugs for synergistic concurrent chemoimmunotherapy against primary and metastatic tumors, along with guided cargo release by PAI in the NIR-II window (Fig. 5).

\section{PTT}

In recent years, PTT, which is a minimally invasive tumor treatment technique, has been developed. This treatment method uses a laser (mostly near-infrared laser) as the external energy source, absorbs nearinfrared light with special photothermal therapeutic 

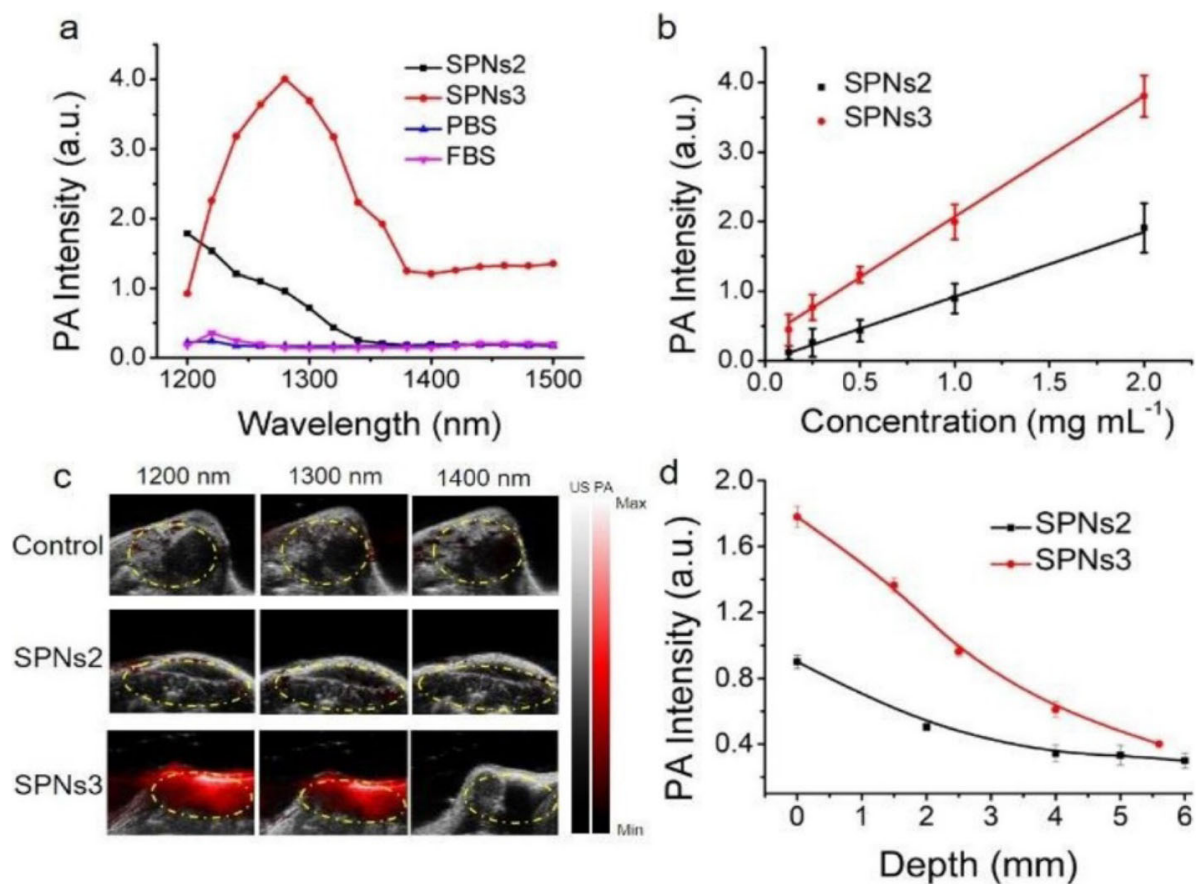

Fig. 4 a PA spectra of $2 \mathrm{mg} / \mathrm{mL}$ SPNs2-3, PBS, and FBS; $\mathbf{b}$ PA signal of SPNs2-3 at various concentrations; $\mathbf{c}$ PA/US co-registered images without injection of SPNs2-3 (control) and with intratumoral injection of $50 \mu \mathrm{L}, 500 \mu \mathrm{g} / \mathrm{mL}$ SPNs2-3 (dashed circles); d Fitted PA signal curves of SPNs2-3 solutions (1 $\mathrm{mg} / \mathrm{mL})$ at various depths in chicken breast tissue [22]

agent, converts the absorbed light energy into heat, causes the temperature of the tumor site to rise, and thus induces cell apoptosis or produces a direct lethal effect on cells. Because near-infrared lasers can penetrate the skin and tissue, effective thermal destruction of deep tumor tissue can be achieved without damaging normal cells. It has been widely used in accurate ablation of tumors with low systemic toxicity. In addition, PAI with high resolution and deep tissue penetration characteristics uses a naturally induced ultrasonic signal based on the photothermal effect of PTT reagent under pulsed near-infrared laser irradiation. The PA signal is primarily determined by photothermal conversion, which is the inherent principle of PTT, making PAI and PTT an ideal combination. PAI and PTT provide useful tools for diagnosis and treatment of diseases. However, current applications of PAI and PTT are greatly limited by their reliance on light sources in the first NIR-I window $(750-1000 \mathrm{~nm})$, which demonstrates shallow tissue penetration depth. This cannot satisfy the requirements of PAI and PTT conversion. NIR-II exogenous PAI contrast agent also exhibits good application prospects for PTT because of its strong absorbance and high conversion efficiency of photothermal spectral analysis [24]. It can greatly reduce the potential damage to normal tissue during PTT. In 2020, Liu et al. [25] reported an innovative $\mathrm{Na}_{\mathrm{x}} \mathrm{MnWO}_{3}$-PEG nanoplatform constructed with protected cathodic $\mathrm{Mn}^{2+}$ for stabilized magnetic resonance imaging (MRI) contrast, demonstrating the ability to guide tumor PTT. The in vitro and in vivo results indicated that $\mathrm{Na}_{\mathrm{x}} \mathrm{MnWO}_{3}$-PEG nanorods could be reliable nanoplatforms for MRI-guided PTT. Moreover, the changed valence of the tungsten element under redox status may contribute to the depletion of intracellular glutathione for further enhanced PTT. We believe that this brand-new concept will offer opportunities for developing more reliable and low-toxicity nanoagents for stable MRI, as well as more specific tumor therapy. In vivo T1-weighted MR images (Fig. 6a) and signals (Fig. 6b) of 4 T1-tumor-bearing mice before and after intravenously (i.v.) injection of NaxMnWO3-PEG nanorods $(40 \mathrm{mg} / \mathrm{kg})$. As shown in Figs. $6 \mathrm{c}$ and d, the PA signal could be detected as early as $1 \mathrm{~h}$ after i.v. injection of NaxMnWO3-PEG $(40 \mathrm{mg} / \mathrm{kg})$, and the signal intensity increased gradually over time and remained high even after $24 \mathrm{~h}$, which was in accordance with the performance in MRI. Not only is PTT effective because it can lead to hyperthermia, it can also achieve photothermal ablation (PTA). Hyperpyrexia refers to the rise of tissue temperature to $42-46^{\circ} \mathrm{C}$, while irreversible PTA can lead to tissue necrosis, which is equivalent to $240 \mathrm{~min}$ at $43^{\circ} \mathrm{C}$ or $1 \mathrm{~s}$ at $54^{\circ} \mathrm{C}$. Yao et al. [26] reported leveraging 


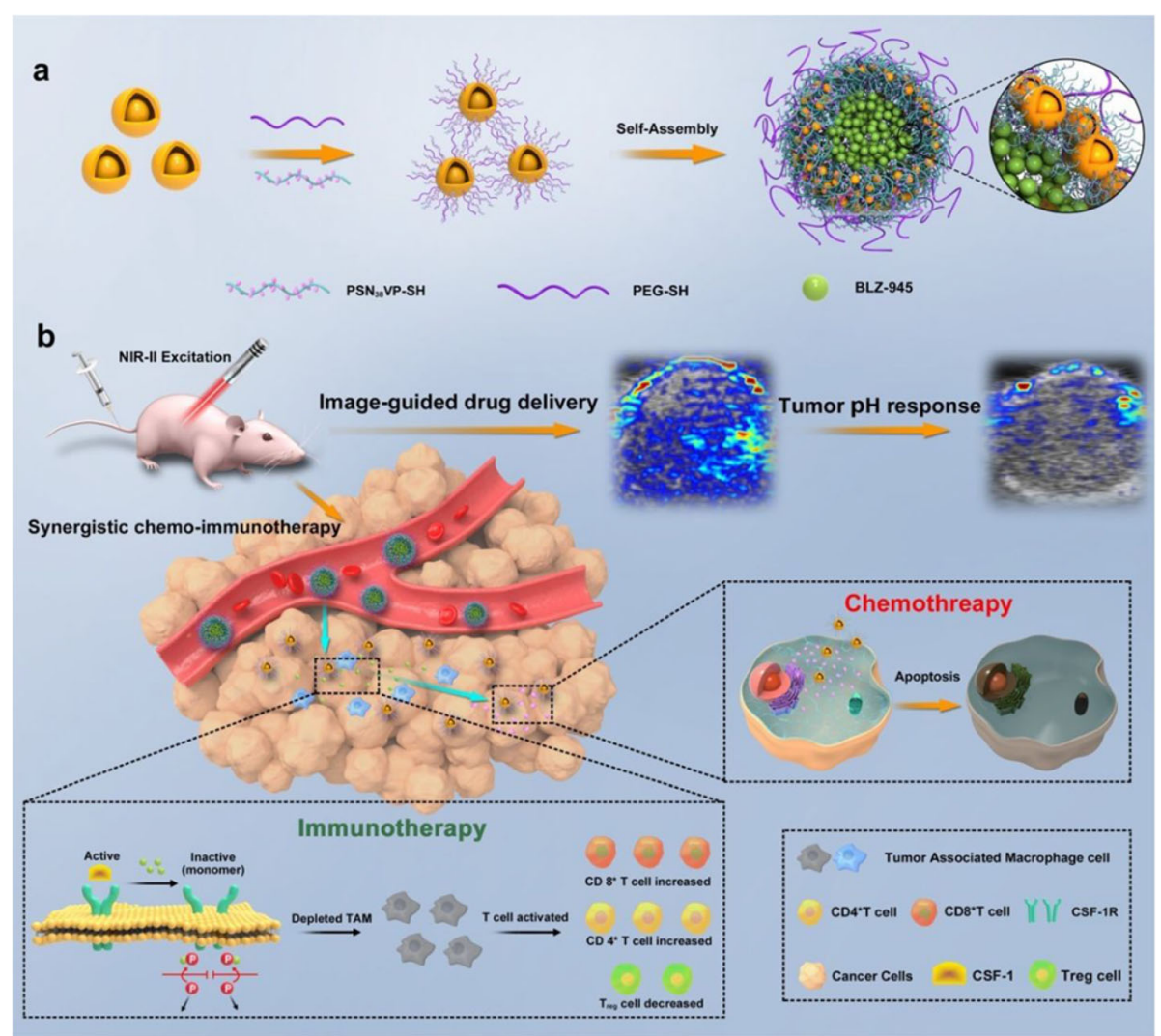

Fig. 5 Preparation of dual-responsive AuNNP@SN ${ }_{38}$ Ve loaded with BLZ-945. The smaller AuNNP@PEG/PSN ${ }_{38} V P$ then penetrated the deeper tumor regions and released the $\mathrm{SN}_{38}$ prodrug in the reductive environment, leading to tumor cell apoptosis. PA imaging in the NIR-II window further enabled drug release monitoring and guided therapy [23]

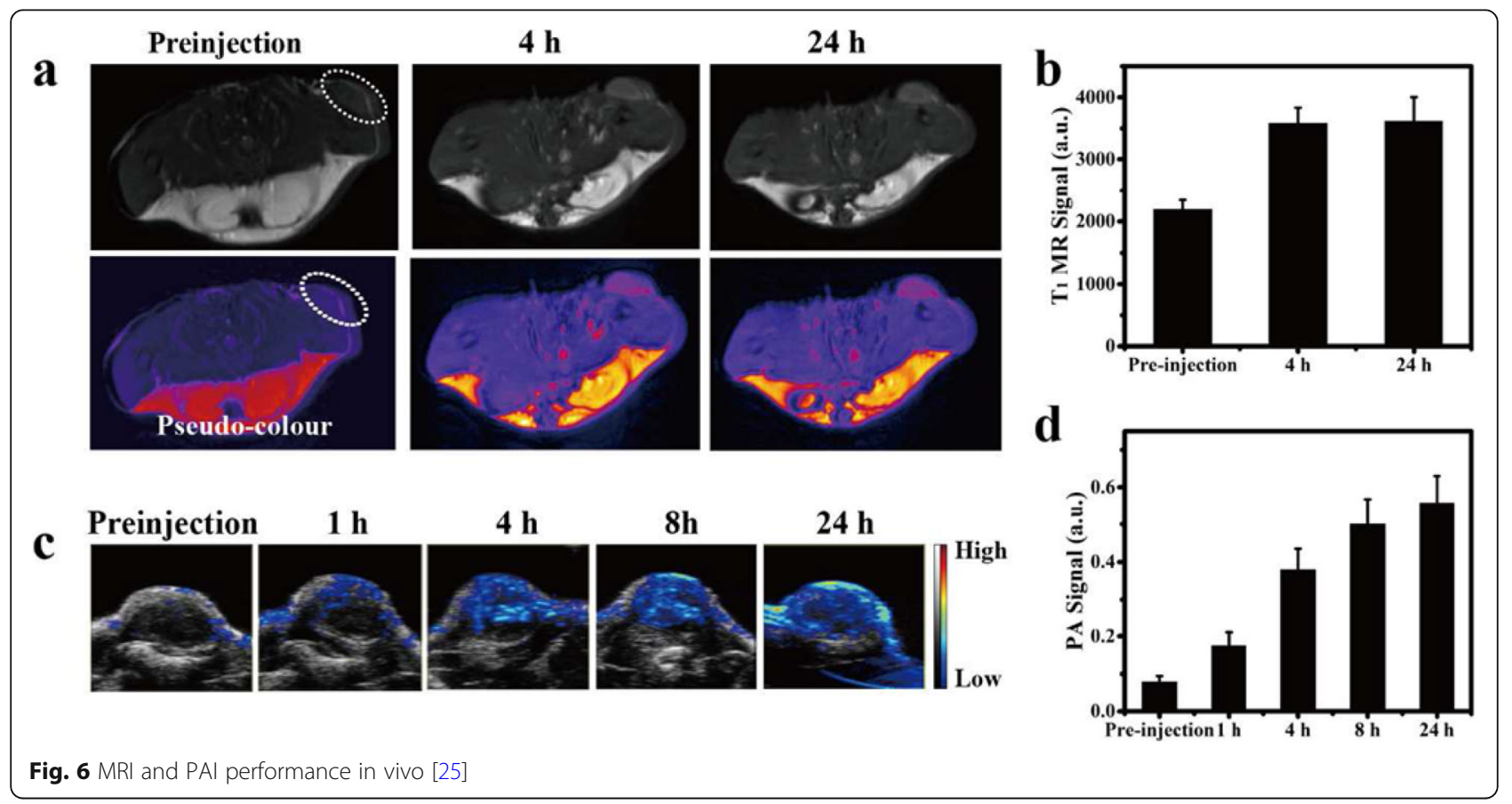




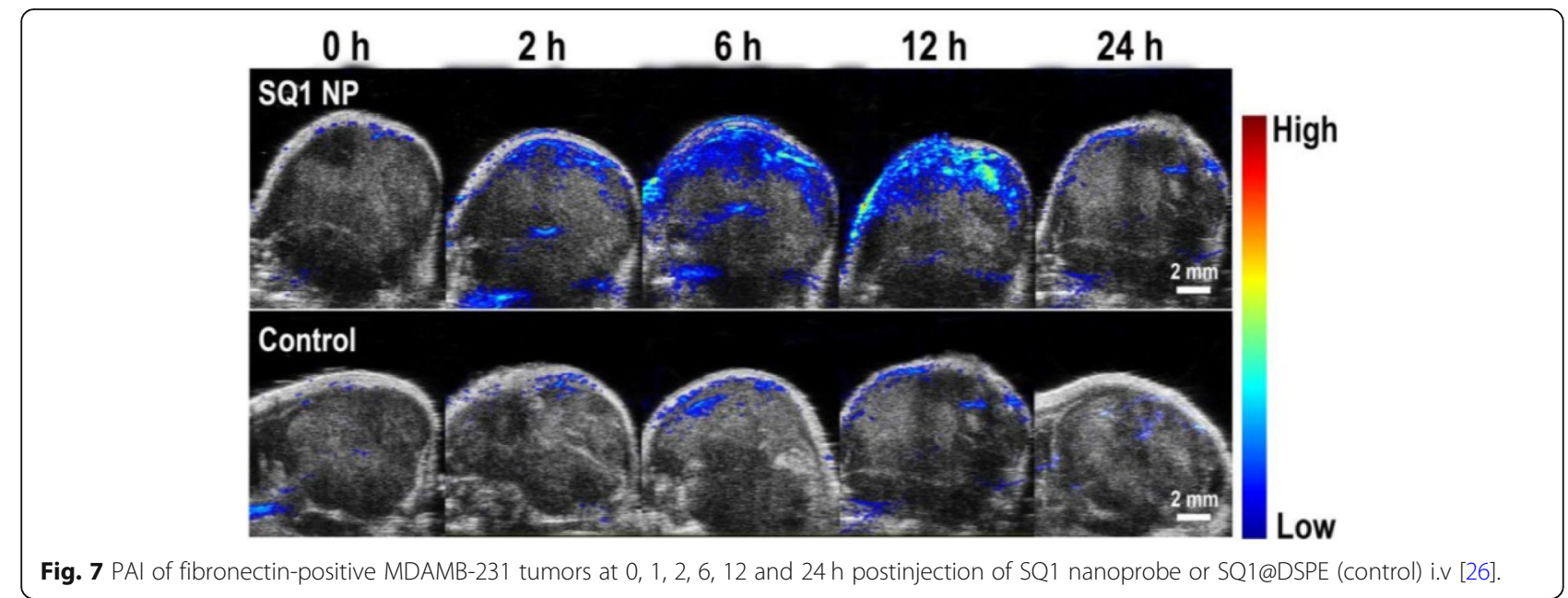

the excellent photothermal conversion capability under NIR irradiation to show a squaraine dye (SQ1) nanoprobe that performed well in both PAI and PTT of solid tumors. These results indicate that SQ1 nanoprobes have significant tumor targeting imaging capabilities.
Moreover, a SQ1 nanoprobe can be used for PTA of tumors. Depending on the photothermal effect of the SQ1 nanoprobe, PAI can be carried out simultaneously (Fig. 7). The PAI signals in the tumor regions increased and reached the maximum value at $12 \mathrm{~h}$.

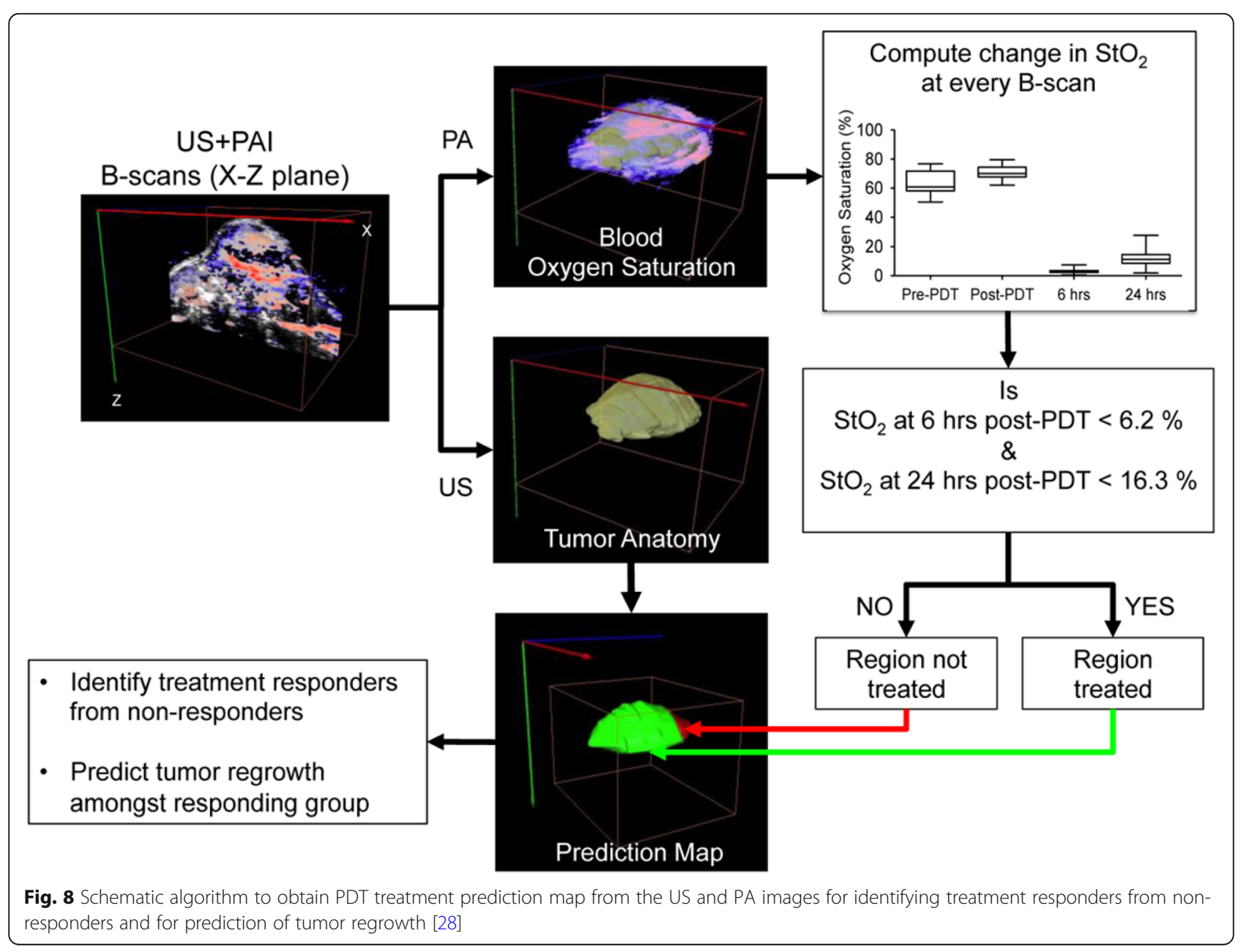




\section{Photodynamic therapy}

Photodynamic therapy (PDT) is a photochemical-based, clinically used technique that produces cytotoxic substances by consuming oxygen, leading to cell death and vessel closure. Vascular damage reduces $\mathrm{StO}_{2}$. This causes a change in blood $\mathrm{StO}_{2}$. PDT as a therapeutic approach, and PAI as a noninvasive, reactive, and disease recurrence monitoring means, are a step toward this goal. Here, the author discussed that $\mathrm{StO}_{2}$ changes measured by PAI after PDT can be used as a detection method to predict the therapeutic effect and tumor recurrence [27]. In 2015, Mallidi et al. [28] investigated whether this change in $\mathrm{StO}_{2}$ measured by PAI post-PDT could act as a surrogate marker for predicting treatment efficacy and tumor recurrence. The findings of this study could possibly be used to guide and monitor several treatment modalities, such as PDT, radiation, and antiangiogenic therapy, that involve a change in $\mathrm{StO}_{2}$. The author devised an algorithm to analyze the $\mathrm{StO}_{2}$ images of recurrent tumors obtained at various time points post-therapy. First, US and PAI B-scans were acquired at step sizes of $0.152 \mathrm{~mm}$ to obtain 3D maps of the anatomy and $\mathrm{StO}_{2}$. At every B-scan, the tumor region was mapped using US imaging and the average $\mathrm{StO}_{2}$ in the region was calculated. If the average $\mathrm{StO}_{2}$ at $6 \mathrm{~h}$ postPDT and $24 \mathrm{~h}$ post-PDT in a particular B-scan frame was less than $6.2 \%$ and $16.3 \%$, respectively, the B-scan region was considered treated and pseudo-colored as green; otherwise, the regions were pseudo-colored red to indicate no treatment (Fig. 8). Tumor hypoxia is the Achilles heel of oxygen-dependent PDT, and tremendous efforts are required to reverse tumor hypoxia. Zhao et al. [29] confirmed that a photosensitizer of chlorine e6 (Ce6)-based self-delivery nanomedicine (ACSN) effectively suppressed oxygen consumption to reverse tumor hypoxia by inhibiting mitochondrial respiration. Benefiting from the synergistic mechanism, an enhanced PDT effect for ACSN was observed regarding the inhibition of tumor growth. This self-delivery system for oxygen-economized PDT could be an appealing clinical strategy for tumor eradication. To verify whether this reduced $\mathrm{O}_{2}$ consumption could relieve tumor hypoxia, 4 T1-tumor-bearing mice were i.v. injected with ACSN for PAI detection. As depicted in Fig. 9a-i, schematic illustration of the proposed mechanism of ACSN for hypoxia remission by mitochondrial complex III inhibition. CLSM images of $4 \mathrm{~T} 1$ cells after being treated with Ce6, ATO, or ACSN and then stained by rhodamine 123 . CLSM images of $4 \mathrm{~T} 1$ cells after being treated with Ce6, ATO, or ACSN in hypoxia and then stained by a Hypoxia/Oxidative Stress Detection kit. Seahorse XF24 Flux analysis of $4 \mathrm{~T} 1$ cells after treatment with Ce6, ATO, or ACSN for $12 \mathrm{~h}$. The basal oxygen consumption rate (OCR), maximal OCR, and ATP production of $4 \mathrm{~T} 1$ cells after various treatments. Schematic illustration of the instrument to measure the $\mathrm{O}_{2}$ consumption of tumor cells. The dissolved $\mathrm{O}_{2}$ in a culture medium of $4 \mathrm{~T} 1$ cells after various treatments. As shown in Fig. 9j, the PA signals gradually increased over time, reflecting the increasing $\mathrm{O}_{2}$ content in the tumors. In particular, the signals peaked within $6 \mathrm{~h}$ and then decreased slightly. The average $\mathrm{StO}_{2}$ was also quantified, as demonstrated in Fig. $9 \mathrm{k}$.

\section{Multi-mode imaging}

Multi-mode imaging is an important development in PAI that integrates a variety of imaging technologies and can provide a variety of types of information about the imaging region to improve the reliability, diversity, and authenticity of scientific research data. The study of PA multimode imaging can be generally divided into two aspects: PA/US imaging has inherent multi-parameter characteristics, which can be used to obtain multiparameter PA/US images.

Examples include PA images of blood $\mathrm{StO}_{2}$, total hemoglobin, deoxygenated hemoglobin, hypoxic hemoglobin, melanin, lipids, and other specific components. In addition, a variety of imaging parameters, such as tissue structure, blood flow velocity, and vascular perfusion, can be extracted by combining PA with ultrasonic signals [30, 31]. In 2019, Meng et al. [32] designed a US-responsive PA imaging probe based on microbubbles (MBs) containing Au NPs for in vivo "backgroundfree" PA imaging. The obtained Au@lip MBs with separated $\mathrm{Au}$ NPs decorated within the lipid shell of MBs show low PA signals under near-infrared (NIR) excitation. Interestingly, under exposure to US pulses, these Au@lip MBs burst to form nanoscale aggregates of Au@lip NPs, which exhibit significantly enhanced NIR PA signals because of their red-shifted surface plasmon resonance. Scheme showing the experimental process. First, mice bearing CT26 tumors were orally treated with Erlotinib (EB) and then i.v. injected with Au@lip MBs for ultrasound imaging and photoacoustic imaging in sequence. Au@lip MBs were used as the contrast agents for both ultrasound and photoacoustic imaging. MBbased US imaging is an established method for studying blood perfusion in selected organs. Different from untreated tumors, in which US signals were detected only in their surrounding regions, strong US signals emerged in tumors immediately after the injection of Au@lip MBs and were dispersed throughout the tumor for mice with EB treatment (Fig. 10 a-c).

In particular, for the tumor image in the untreated group with Au@lip MB injection, a large blood vessel happened to show up in the original PA image. By background subtraction, signals from this blood vessel could be completely eliminated to avoid false imaging results 


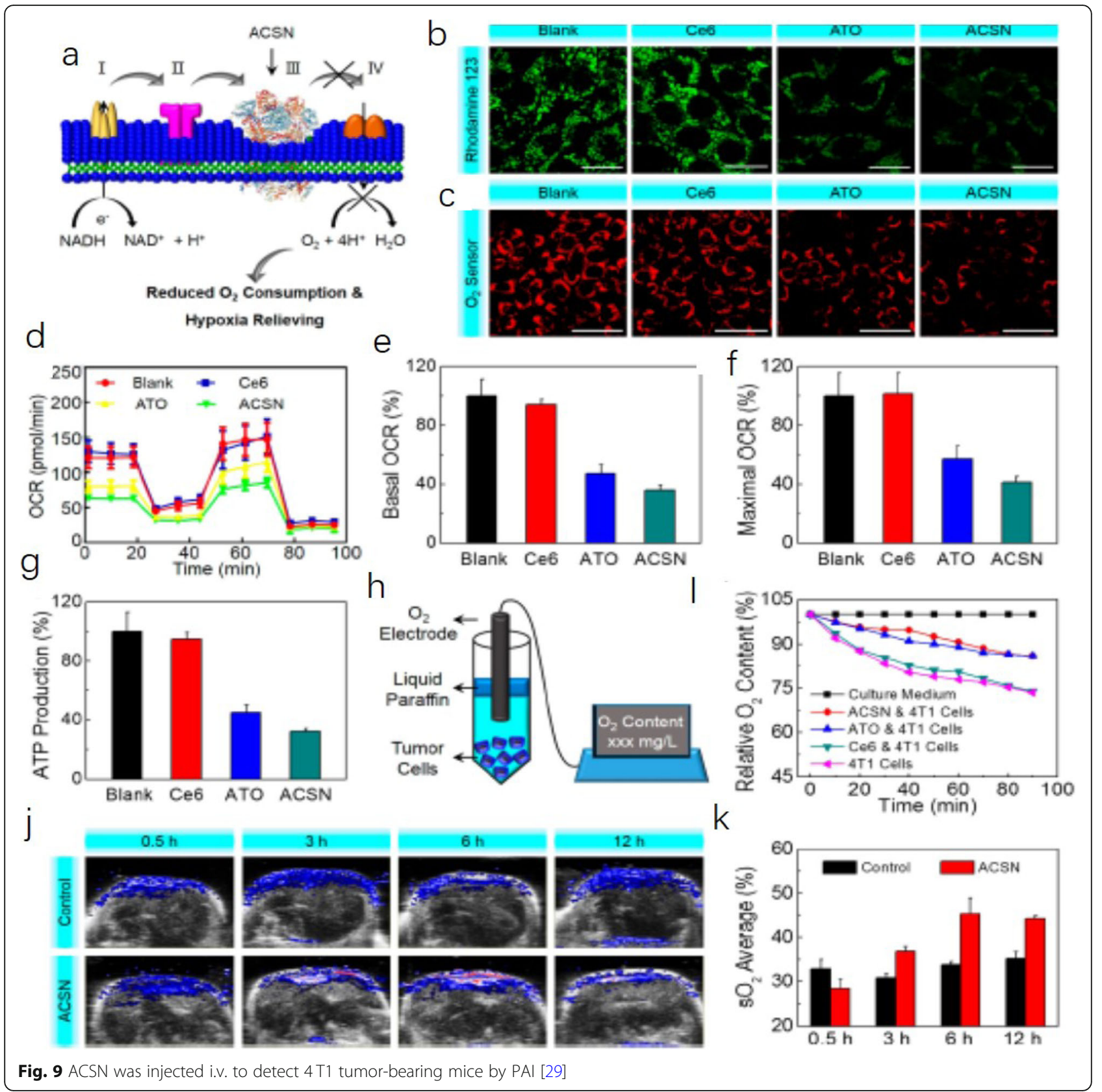

owing to the background interference (Fig. $10 \mathrm{~d}$ and j). Compared to tumors on untreated mice, those on mice after erlotinib treatment showed a significantly increased accumulation of Au@lip MBs (Fig. $10 \mathrm{~d}$ and h). In contrast, when the conventional probe $\mathrm{CuS}$ NPs were used, it became difficult to identify whether the observed PA signals in the tumor were from the NPs or just the background from tumor blood vessels (Fig. $10 \mathrm{~d}-\mathrm{f}$ ). The PA model was well combined with the US microvesicle imaging model to obtain the PA signal data of tumor vascular perfusion. Conversely, multi-mode contrast agents can be applied to a variety of imaging systems, such as
PAI, nuclear magnetic imaging, computed tomography, optical imaging, and ultrasonic imaging, and have been used to improve the accuracy of scientific research diagnosis [33]. Lemaster et al. [34] in a 2019 study provided details of a synthetic melanin-based contrast agent for PAI and MRI. The most important finding is that the PA intensity increased dramatically upon incorporation of metal ions into polydopamine-based nanoparticles. Chelation is known to increase the biocompatibility of Gd-based contrast agents, which are clinically used in MRI. We used the Gd (III)-enhanced PA signal to image stem cells in vivo by coupling this modality with MRI. 


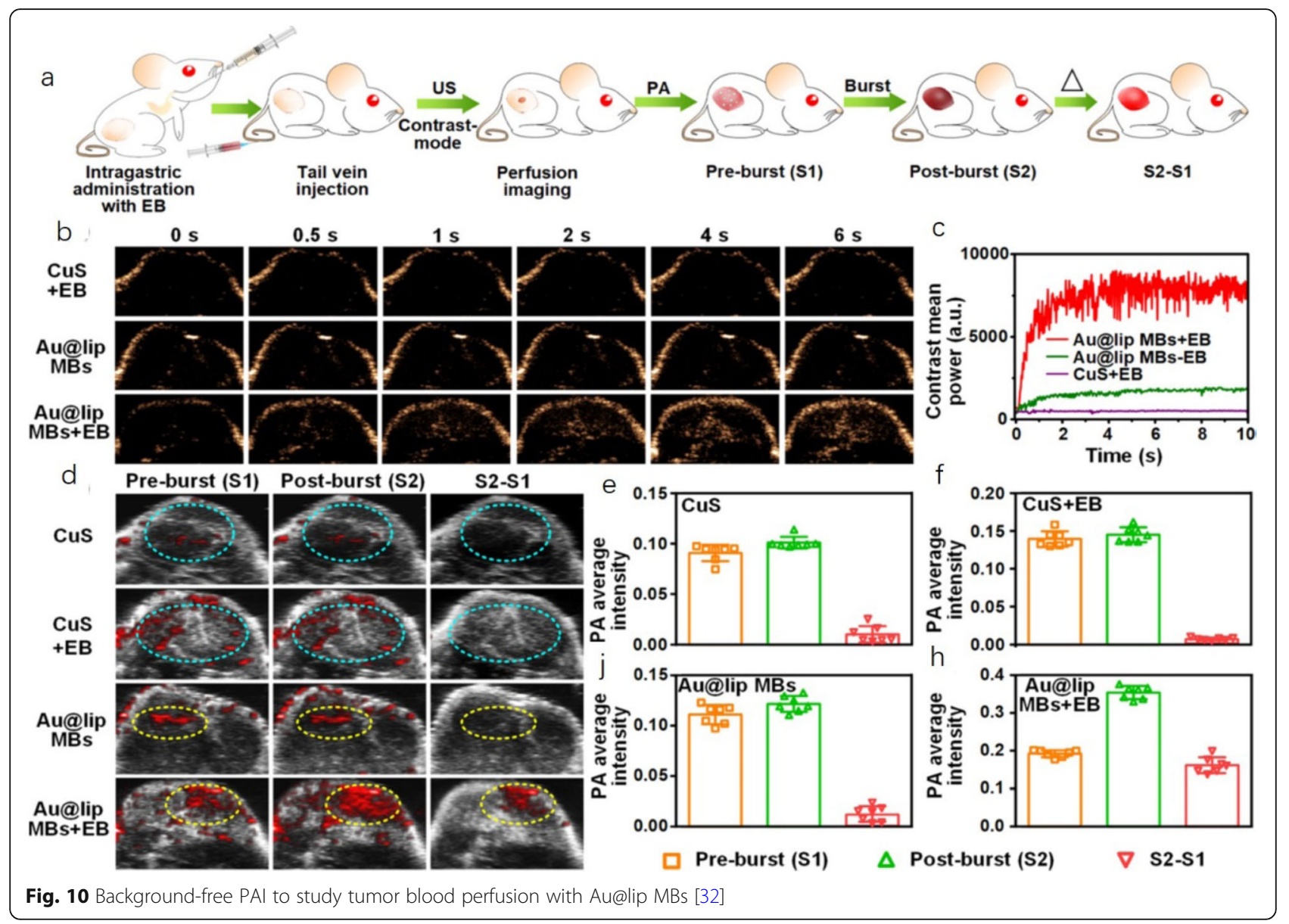

The labeled stem cells still expressed stem cell surface markers and continued to proliferate. In vivo experiments using 500,000 cells labeled with gadoliniumloaded synthetic melanin nanoparticles [Gd (III)-SMNP] particles were performed in mice (Fig. 11). Bone marrow mononuclear cells have been found to promote heart function and neovascularization after myocardial infarction via intramyocardial injection delivery. Echocardiograms pre- and immediately post-injection are also shown in Fig. 11, which indicate that the PA signal increased 64-fold.

\section{Summary and outlook}

PAI technology combines the advantages of optical imaging and acoustic imaging, making the detection deeper, more accurate, and safer [35].

PAI employs non-ionizing laser radiation, which has higher biological safety than conventional CT imaging, MRI imaging, and other types of ionizing radiation. Therefore, it has become one of the most rapidly developing new biomedical imaging technologies. In particular, the multimode imaging system that integrates photoacoustic and ultrasonic imaging has emerged in recent years, as PAI and ultrasonic imaging demonstrate natural complementarity, and the system has a high degree of compatibility. The multimode real-time imaging system is realized by the organic integration of the two, to obtain imaging information and better meet the needs of scientific research for accurate detection and early diagnosis. The emergence of NIR-II PAI $(1000 \mathrm{~nm}-$ $2000 \mathrm{~nm}$ ) has overcome the background interference and spontaneous fluorescence associated with NIR-I $(650 \mathrm{~nm}-900 \mathrm{~nm})$, thus improving the sensitivity, spatial resolution, and deeper tissue penetration depth of PAI.

Because of the inherent characteristics of PPT and PDT, PAI is the most effective detection method. PPT and PDT demonstrate good biological compatibility to avoid potential toxicity caused by their retention in the body, and high conversion efficiency to avoid damage to normal tissues. Therefore, they show good application prospects in present research. Multi-mode imaging and real-time imaging systems have become the focus of PA research and an important development trend for the future. In particular, the combination of PA and ultrasonic 


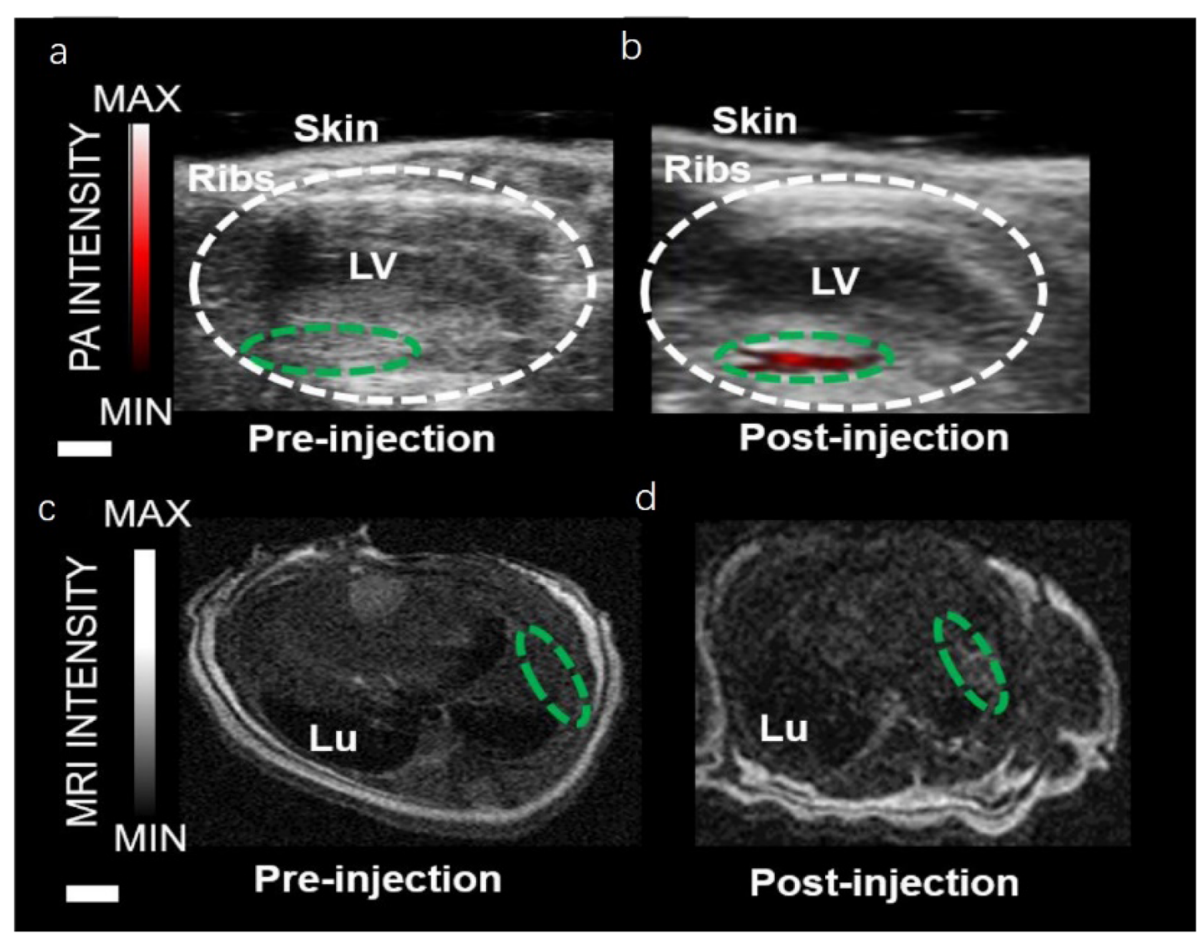

Fig. 11 PAI and MRI of Gd (III)-SMNP implanted into mouse hearts [34]

imaging can be used as an acoustic signal detection system. Moreover, the introduction of high-frequency technology into US and PAI can advance basic research. Meanwhile, multi-mode real-time imaging systems integrated with PA/US technology can provide more information, improve the accuracy of results, and better meet the needs of preclinical research for early and accurate diagnosis.

\section{Abbreviations}

PA: Photoacoustic; US: Ultrasound; PA/US: Photoacoustic/ultrasound; PANPS: Photoacoustic properties of nanoparticles; hESC-CM: Human embryonic stem cell—cardio myocytes; SP: Semiconductor polymer; tHI: Transient hypoxia-ischemia; RICA: Right internal carotid artery; RCCAO: Right carotid artery occlusion; $\mathrm{StO}_{2}$ : Oxygen saturation; PAl: Photoacoustic imaging; PTT: Photothermal therapy; NIR-II: The second near-infrared; SPNs: Semiconducting polymer nanoparticles;

PTA: Photothermal ablation; PDT: Photodynamic therapy; MRI: Magnetic resonance imaging; SQ1: Squaraine nanoprobe; ACSN: A photosensitizer of chlorine e6 (Ce6)-based self-delivery nanomedicine; MBs: Microbubbles; Au NPs: Gold nanoparticles; Gd (III)-SMNP: Gadolinium-loaded synthetic melanin nanoparticles; OCR: Oxygen consumption rate; i.v.: Intravenously

\section{Acknowledgments}

The authors want to acknowledge the technical help of FUJIFILM

VisualSonics Department.

\section{Authors' contributions}

SW, YZ and YX approved the final versions of tables and figures and got the authorization of each author for the submission after their revision; the authors read and approved the final manuscript.

\section{Funding}

Not applicable.
Availability of data and materials

All data analysed during this study are included in this published article.

\section{Competing interests}

The authors declare that they have no competing interests.

\section{Author details}

${ }^{1}$ VisualSonics Business Department, FUJIFILM (China) Investment Co. Ltd., Beijing 100026, China. ${ }^{2}$ VisualSonics Business Department, FUJIFILM (China) Investment Co. Ltd., Shanghai 200120, China.

Received: 9 June 2020 Accepted: 2 October 2020 Published online: 21 October 2020

\section{References}

1. Zackrisson S, van de Ven SMWY, Gambhir SS (2014) Light in and sound out: emerging translational strategies for photoacoustic imaging. Cancer Res 74(4):979-1004. https://doi.org/10.1158/0008-5472.CAN-13-2387

2. Needles A, Heinmiller A, Sun J, Theodoropoulos C, Bates D, Hirson D et al (2013) Development and initial application of a fully integrated photoacoustic micro-ultrasound system. IEEE Trans Ultrason Ferroelectr Freq Control 60(5):888-897. https://doi.org/10.1109/TUFFC.2013.2646

3. Nam SY, Chung E, Suggs LJ, Emelianov SY (2015) Combined ultrasound and photoacoustic imaging to noninvasively assess burn injury and selectively monitor a regenerative tissue-engineered construct. Tissue Eng Part C Methods 21(6):557-566. https://doi.org/10.1089/ten.tec.2014.0306

4. Huang DD, Qiu Q, Lin WZ, Liu JY, Huang YL, Zhao QL (2019) Recent advances in biomedical applications of dual-modality photoacoustic/ ultrasound imaging technology. J Light Scatt 31(1):1-10

5. Zafar H, Breathnach A, Subhash HM, Leahy MJ (2015) Linear-array-based photoacoustic imaging of human microcirculation with a range of high frequency transducer probes. J Biomed Opt 20(4):051021. https://doi.org/10. 1117/1.JBO.20.5.051021

6. Gray JP, Dana N, Dextraze KL, Maier F, Emelianov S, Bouchard RR (2016) Multi-wavelength photoacoustic visualization of high intensity focused ultrasound lesions. Ultrason Imaging 38(1):96-112. https://doi.org/10.1177/ 0161734615593747 
7. Liu M, Zhang XY, Zhou TT (2018) Application and research progress of highfrequency ultrasound technology for small animals. Chin J Hemorheol 28(4): 492-496

8. Luke GP, Emelianov SY (2015) Label-free detection of lymph node metastases with US-guided functional photoacoustic imaging. Radiology 277(2):435-442. https://doi.org/10.1148/radiol.2015141909

9. Gerling M, Zhao Y, Nania S, Norberg KJ, Verbeke CS, Englert B et al (2014) Real-time assessment of tissue hypoxia in vivo with combined photoacoustics and high-frequency ultrasound. Theranostics 4(6):604-613. https://doi.org/10.7150/thno.7996

10. Nam SY, Ricles LM, Suggs LJ, Emelianov SY (2012) In vivo ultrasound and photoacoustic monitoring of mesenchymal stem cells labeled with gold nanotracers. PLoS One 7(5):e37267. https://doi.org/10.1371/journal.pone. 0037267

11. Talukdar Y, Avti P, Sun J, Sitharaman B (2014) Multimodal ultrasoundphotoacoustic imaging of tissue engineering scaffolds and blood oxygen saturation in and around the scaffolds. Tissue Eng Part C Methods 20(5): 440-449. https://doi.org/10.1089/ten.tec.2013.0203

12. Bok TH, Hysi E, Kolios MC (2016) Simultaneous assessment of red blood cell aggregation and oxygen saturation under pulsatile flow using highfrequency photoacoustics. Biomed Opt Express 7(7):2769-2780. https://doi. org/10.1364/BOE.7.002769

13. Kim J, Park S, Jung Y, Chang S, Park J, Zhang YM et al (2016) Programmable real-time clinical photoacoustic and ultrasound imaging system. Sci Rep 6: 35137. https://doi.org/10.1038/srep35137

14. Garcia-Uribe A, Erpelding TN, Krumholz A, Ke HX, Maslov K, Appleton C et al (2015) Dual-modality photoacoustic and ultrasound imaging system for noninvasive sentinel lymph node detection in patients with breast cancer. Sci Rep 5:15748. https://doi.org/10.1038/srep15748

15. Daoudi K, van den Berg PJ, Rabot O, Kohl A, Tisserand S, Brands P et al (2014) Handheld probe integrating laser diode and ultrasound transducer array for ultrasound/photoacoustic dual modality imaging. Opt Express 22(21):26365-26374. https://doi.org/10.1364/OE.22.026365

16. Sun YY, Li YK, Wali B, Li YC, Lee J, Heinmiller A et al (2015) Prophylactic edaravone prevents transient hypoxic-ischemic brain injury: implications for perioperative neuroprotection. Stroke 46(7):1947-1955. https://doi.org/10. 1161/STROKEAHA.115.009162

17. Qin XL, Chen HD, Yang HX, Wu HD, Zhao X, Wang HY et al (2018) Photoacoustic imaging of embryonic stem cell-derived cardiomyocytes in living hearts with ultrasensitive semiconducting polymer nanoparticles. Adv Funct Mater 28(1):1704939. https://doi.org/10.1002/adfm.201704939

18. Wang JP, Sun JY, Wang YH, Chou T, Zhang Q, Zhang BL et al (2020) Gold nanoframeworks with mesopores for raman-photoacoustic imaging and photo-chemo tumor therapy in the second near-infrared biowindow. Adv Funct Mater 30(9):1908825. https://doi.org/10.1002/adfm.201908825

19. Sangha GS, Phillips EH, Goergen CJ (2017) In vivo photoacoustic lipid imaging in mice using the second near-infrared window. Biomed Opt Express 8(2):736-742. https://doi.org/10.1364/BOE.8.000736

20. Wang B, Karpiouk A, Yeager D, Amirian J, Litovsky S, Smalling R et al (2012) Intravascular photoacoustic imaging of lipid in atherosclerotic plaques in the presence of luminal blood. Opt Lett 37(7):1244-1246. https://doi.org/10. 1364/OL.37.001244

21. Miao QQ, Pu KY (2018) Organic semiconducting agents for deep-tissue molecular imaging: second near-infrared fluorescence, self-luminescence, and photoacoustics. Adv Mater 30(49):1801778. https://doi.org/10.1002/ adma.201801778

22. Zhang WS, Sun XL, Huang T, Pan XX, Sun PF, Li JW et al (2019) 1300 nm absorption two-acceptor semiconducting polymer nanoparticles for NIR-II photoacoustic imaging system guided NIR-II photothermal therapy. Chem Commun 55(64):9487-9490. https://doi.org/10.1039/C9CC04196F

23. Zhu R, Su LC, Dai JY, Li ZW, Bai SM, Li QQ et al (2020) Biologically responsive plasmonic assemblies for second near-infrared window photoacoustic imaging-guided concurrent chemo-immunotherapy. ACS Nano 14(4):3991-4006. https://doi.org/10.1021/acsnano.9b07984

24. Lyu Y, Li JC, Pu KY (2019) Second near-infrared absorbing agents for photoacoustic imaging and photothermal therapy. Small Methods 3(11): 1900553. https://doi.org/10.1002/smtd.201900553

25. Liu Y, Wu SM, Liu YY, Zhang H, Zhang M, Tang ZM et al (2020) Cathodic protected $\mathrm{Mn} 2+$ by NaxWO3 nanorods for stable magnetic resonance imaging-guided tumor photothermal therapy. Biomaterials 234:119762. https://doi.org/10.1016/j.biomaterials.2020.119762
26. Yao DF, Wang YS, Zou RF, Bian KX, Liu P, Shen SZ et al (2020) Molecular engineered squaraine nanoprobe for NIR-II/photoacoustic imaging and photothermal therapy of metastatic breast cancer. ACS Appl Mater Interfaces 12(4):4276-4284. https://doi.org/10.1021/acsami.9b20147

27. Eisenbrey JR, Merton DA, Marshall A, Liu JB, Fox TB, Sridharan A et al (2015) Comparison of photoacoustically derived hemoglobin and oxygenation measurements with contrast-enhanced ultrasound estimated vascularity and immunohistochemical staining in a breast cancer model. Ultrason Imaging 37(1):42-52. https://doi.org/10.1177/0161734614527435

28. Mallidi S, Watanabe K, Timerman D, Schoenfeld D, Hasan T (2015) Prediction of tumor recurrence and therapy monitoring using ultrasound-guided photoacoustic imaging. Theranostics 5(3):289-301. https://doi.org/10.7150/ thno.10155

29. Zhao LP, Zheng RR, Chen HQ, Liu LS, Zhao XY, Liu HH et al (2020) Selfdelivery nanomedicine for O2-economized photodynamic tumor therapy. Nano Lett 20(3):2062-2071. https://doi.org/10.1021/acs.nanolett.0c00047

30. Tao C, Xiang SP (2016) Multi-modal imaging system based on photoacoustic-ultrasound cooperation. Chin J Med Phys 33(12):1240-1244

31. Bar-Zion A, Yin M, Adam D, Foster S (2016) Functional flow patterns and static blood pooling in tumors revealed by combined contrast-enhanced ultrasound and photoacoustic imaging. Cancer Res 76(15):4320-4331. https://doi.org/10.1158/0008-5472.CAN-16-0376

32. Meng ZQ, Zhou XF, She JL, Zhang YJ, Feng LZ, Liu Z (2019) Ultrasoundresponsive conversion of microbubbles to nanoparticles to enable background-free in vivo photoacoustic imaging. Nano Lett 19(11):81098117. https://doi.org/10.1021/acs.nanolett.9b03331

33. de la Zerda A, Liu Z, Bodapati S, Teed R, Vaithilingam S, Khuri-Yakub BT et al (2010) Ultrahigh sensitivity carbon nanotube agents for photoacoustic molecular imaging in living mice. Nano Lett 10(6):2168-2172. https://doi. org/10.1021/nl100890d

34. Lemaster JE, Wang Z, Hariri A, Chen F, Hu ZY, Huang YR et al (2019) Gadolinium doping enhances the photoacoustic signal of synthetic melanin nanoparticles: a dual modality contrast agent for stem cell imaging. Chem Mater 31(1):251-259. https://doi.org/10.1021/acs.chemmater.8b04333

35. Bouchard R, Sahin O, Emelianov S (2014) Ultrasound-guided photoacoustic imaging: current state and future development. IEEE Trans Ultrason Ferroelectr Freq Control 61(3):450-466. https://doi.org/10.1109/TUFFC.2014.2930

\section{Publisher's Note}

Springer Nature remains neutral with regard to jurisdictional claims in published maps and institutional affiliations.

\section{Submit your manuscript to a SpringerOpen ${ }^{\circ}$ journal and benefit from:}

- Convenient online submission

- Rigorous peer review

- Open access: articles freely available online

High visibility within the field

- Retaining the copyright to your article

Submit your next manuscript at $\boldsymbol{\sim}$ springeropen.com 